\title{
The surface properties of small asteroids from thermal-infrared observations
}

\author{
Alan W. Harris \\ DLR Institute of Planetary Research, Rutherfordstrasse 2, 12489 Berlin, Germany \\ email: alan.harris@dlr.de
}

\begin{abstract}
While the physical characterization of near-Earth objects (NEOs) is progressing at a much slower rate than that of discovery, a substantial body of thermal-infrared data has been gathered over the past few years. A wide variety of taxonomic classes in the NEO population have now been sampled by means of thermal-infrared spectrophotometric observations. The resulting albedo information, together with the distribution of taxonomic types from spectroscopic investigations and the rapidly increasing catalog of orbits and absolute magnitudes derived from NEO search programs, such as LINEAR, facilitates more accurate estimates of the size distribution of the NEO population and the magnitude of the impact hazard. Despite our rapidly increasing knowledge of the NEO population, many questions and uncertainties remain, such as: How does the albedo distribution of NEOs compare with that of main-belt asteroids, and does space weathering play a role? How does the surface structure and regolith coverage of NEOs vary with size and taxonomic type? What fraction of NEOs are extinct comets? A property of particular interest is the surface thermal inertia of small asteroids, which is an indicator of the presence or lack of a thermally-insulating surface layer. Large asteroids can accumulate regolith, but can very small asteroids retain thermally-insulating collisional debris or at least a dust layer? Knowledge of thermal inertia is important for accurate calculations of the Yarkovsky effect, which can significantly influence the orbital evolution of potentially hazardous NEOs, and for the design of instruments for lander missions. Contrary to earlier expectations, evidence appears to be accumulating that even sub-kilometer asteroids often have a significant thermally-insulating surface layer. Recent results from thermal-infrared investigations of NEOs are reviewed and implications for the surface properties of small asteroids discussed.
\end{abstract}

Keywords. Asteroids, near-Earth objects, physical properties

\section{Introduction}

Whatever method of investigation is chosen, whether it be in-situ measurements from a spacecraft or remote astronomical observations, the primary physical information we obtain about a target asteroid normally relates to its surface properties and shape. While we can deduce some information on bulk properties and internal structure from in-situ techniques such as radio science, radar and seismic tomography, and the study of binary systems, most observational data, whether optical, infrared, or radar, provide direct information only on external characteristics. It is therefore important to study the range of surface characteristics present among asteroids and determine how these relate to properties such as size, internal structure and mineralogical composition, and the history of the object, in order to maximize the amount of information that can be extracted about a body as a whole from the observational data.

Even basic parameters, such as size and albedo, are notoriously difficult to determine accurately, especially in the case of small asteroids, in particular near-Earth objects (NEOs), which often have very irregular shapes and surface properties that differ widely from one object to another. Most estimates of the sizes of asteroids are based on absolute 
visual magnitudes determined from photometric data and orbital information, together with an assumed geometric albedo, pv. Since the observed range of asteroid albedos is about $2 \%-60 \%$, estimates of diameter derived on the assumption of a typical albedo, e.g. $15 \%$, can be in error by a factor of 2 or more (for a given $\mathrm{H}$-value diameter is proportional to $\left.p_{v}^{-0.5}\right)$. Clearly, for many applications, including studies of the size distribution of asteroid populations, more accurate determinations of size are required.

In some cases it has been possible to determine the sizes of asteroids directly, e.g. from rendezvous or fly-by missions, occultation measurements, or direct imaging by astronomical telescopes. In these cases the known size and the $\mathrm{H}$-value can be combined to derive a relatively reliable value of albedo. However, the vast majority of cataloged albedos have been derived indirectly by means of optical and thermal-infrared observations and the use of a thermal model. Results to date have shown that the albedo distribution of the NEO population appears to be broadly similar to that of the observed main-belt population but there may be some differences in detail. There is evidence that the albedo ranges associated with some spectral classes or taxonomic types in the NEO population are broader than the albedo ranges associated with the same types in the main-belt population. Observations of NEOs sample smaller and younger objects, in general, than observations made to date of main-belt objects, so size- and/or age-dependent phenomena such as the presence, or lack of, impact debris (regolith) and space weathering may offer explanations for apparent differences in the albedo distributions of the NEO and main-belt populations. Furthermore, while most NEOs are thought to be collisional fragments from the main belt, recent studies have shown that at least several percent of the NEO population may have a cometary origin.

Reliable modeling of scattered solar and thermally emitted radiation from small asteroids is very difficult and the interpretation of observational data is prone to error due to the use of techniques that over-simplify the physical description of the objects. Models based on a simplified surface temperature distribution and spherical geometry are often unavoidable due to the lack of information on the physical characteristics of the object. However, the rapidly increasing body of observational data has encouraged the development of sophisticated thermophysical models that take account of shape, spin-axis orientation, thermal inertia and surface roughness. Shape models derived from lightcurve inversion techniques or extensive radar observations can be used as the basis of a thermophysical model. Given the rotation rate and spin-axis orientation, model parameters such as thermal inertia, crater geometry and the surface density of craters can be varied to give the best fit of the model thermal-infrared fluxes to observational data taken at different wavelengths and solar phase angles. Recent results for a number of well-studied asteroids are indicative of marked differences between the thermal characteristics of large main-belt asteroids and NEOs. One area in which such results are of crucial importance is the study of the orbital evolution of NEOs, especially potentially hazardous objects (PHOs). Due to the momentum they carry, the asymmetric emission of thermal photons can cause significant drift in the orbit of a PHO over long time scales and therefore influence the long-term evolution of impact probabilities. For accurate calculations of the orbital drift, resulting from what has become known as the Yarkovsky effect, knowledge of the surface thermal properties of the asteroid is required.

\section{Thermal modeling}

\subsection{Models based on spherical geometry}

The optical brightness of an asteroid depends on the product of its geometric albedo and projected area. While these parameters cannot be individually determined from optical 
photometry alone, if observations of the optical brightness can be combined with measurements of the objects thermal emission, both its albedo and size can be individually derived.

Thermal-infrared measurements have provided the vast majority of asteroid albedo determinations to date. However, in the case of small asteroids, in particular NEOs, complications can arise due to their irregular shapes, the apparent wide range of surface properties, presumably reflecting the presence or absence of a dusty, insulating regolith (small objects may have insufficient gravity to retain collisional debris), and the fact that they are often observed at large solar phase angles. The problem of irregular shape can be largely overcome by combining thermal-infrared observations with lightcurve-tracing optical photometry obtained at about the same time. The optical photometry allows the infrared fluxes to be corrected for rotational variability.

If no physical data are available for an observed asteroid, one has little choice but to adopt a simple thermal model based on spherical geometry. The most common example is the standard thermal model (STM) described by Lebofsky et al. (1986). The STM was designed for use with large main-belt asteroids and incorporates parameters that apply to asteroids having low thermal inertia and/or slow rotation observed at low solar phase angles. In order to extend the applicability of the STM to objects that may have significant thermal inertia and rapid rotation, such as NEOs, Harris (1998) considered a modified approach, the near-Earth asteroid thermal model (NEATM), in which the model temperature distribution is adjusted via the parameter $\eta$ to force consistency with the observed apparent color temperature of the asteroid, which depends on thermal inertia, surface roughness and spin vector (the value of $\eta$ is kept constant in the STM, in which it was originally introduced to take account of the beaming effect at low phase angles due to surface roughness).

In the NEATM and the STM the surface temperature distribution is given by

$$
T(\theta)=T(0) \cos ^{1 / 4} \theta
$$

where $\theta$ is the angular distance from the sub-solar point. For surface elements not visible to the observer at the given phase angle, or on the night side, $T(\theta)$ is set to zero. $T(0)$, the sub-solar temperature, is given by

$$
T(0)=[1-A) S /(\eta \varepsilon \sigma)]^{1 / 4}
$$

where $A$ is the bolometric Bond albedo, $S$ the solar flux at the asteroid, $\eta$ the beaming parameter, $\varepsilon$ the emissivity, and $\sigma$ the Stefan-Boltzmann constant. In the NEATM the beaming parameter is treated as a variable for the purposes of fitting the model continuum to the observed thermal-IR fluxes.

The NEATM requires flux measurements at a minimum of two wavelengths (preferably several, widely spaced around the thermal continuum peak in the range $5-20 \mu \mathrm{m}$ ), whereas for use of the STM only one is required. A further difference between the NEATM and the STM is the treatment of the thermal-infrared phase effect: the STM uses an empirical phase coefficient, typically $0.01 \mathrm{mag} / \mathrm{deg}$, derived from observations of main-belt asteroids at solar phase angles below $30^{\circ}$. Since NEOs are often observed at larger phase angles, the NEATM takes account of the phase angle by means of a numerical integration of the observable thermal emission from that part of the surface of the spherical model illuminated by the Sun. For asteroids with STM-type characteristics observed at low phase angles the results from the STM and the NEATM are very similar (as can be demonstrated by application of both models to the IRAS flux data from which the IRAS Minor Planet Survey results (Tedesco 1992) are derived - see Walker 2003, and http://www.mira.org/research/irasIntro.htm). 
The NEATM is a relatively simple and practical approach to the derivation of diameters and albedos of asteroids in general (not just NEOs!) applicable to the majority of observational circumstances. However, as in the case of the STM, thermal emission from the night side of the object is not accounted for in the model, which is therefore expected to lead to overestimation of diameters and underestimation of albedos for objects with large thermal inertias observed at large solar phase angles. In such cases, use of a simple model with a longitude-independent temperature distribution, often called the fast-rotating or isothermal-latitude model (FRM/ILM, Lebofsky \& Spencer 1989), may be preferable ( $\operatorname{see} \S 6)$.

The above discussion of the commonly used thermal models is a very brief summary for the purposes of this review. For more detailed discussions of the NEATM and other thermal models outlined here see Harris (1998), Delbó \& Harris (2002), Harris \& Lagerros (2002), and Delbó et al. (2003), and references therein. Delbó \& Harris (2002) give the mathematical expressions for calculating the wavelength-dependent observable thermalinfrared fluxes for all three models.

\subsection{Thermophysical models}

Improvements in computing power in recent years have paved the way for the development of sophisticated thermophysical models that incorporate physical descriptions of thermal inertia and surface roughness. Clearly such models should provide more reliable results than the simpler models described above but they require some prior information about the asteroid. For example, information on shape and spin-axis orientation may be available from lightcurve or radar observations. If extensive lightcurve data are available then it may be possible to construct a shape model of an asteroid from lightcurve inversion techniques (see, for example, Kaasalainen et al. 2004, and references therein) that can be used as the basis of the thermophysical model. Typically, the surface of the asteroid is modeled by means of a convex mesh of several thousand facets. Hemispheres, or sections thereof, can be added to the facets to model the effects of shadowing and multiple reflections of solar and thermally emitted radiation in craters. Unfortunately most shape models currently available do not include intermediate-scale topographical structure, so a more realistic representation of surface roughness is normally not possible. The lack of information on surface structure is a serious problem in the case of NEOs, which are often observed at large phase angles at which shadowing effects are more significant and measured fluxes are presumably strongly dependent on surface roughness.

The temperature of each facet can be determined by applying a simple one-dimensional vertical heat-transfer algorithm (e.g. that of Spencer, Lebofsky \& Sykes 1989) assuming values of albedo and thermal inertia. By summing the contributions from each facet visible to the observer the total model thermal-infrared flux can be compared to flux measurements made at the telescope, and model parameters adjusted to give the best overall fit to the observational data, thereby constraining the physical properties of the observed asteroid.

The detailed thermophysical modeling of main-belt asteroids has been pioneered by Johan Lagerros, who has published a series of detailed papers on various aspects of the field. For an overview and list of references see Harris \& Lagerros (2002). Sophisticated thermophysical modeling based on the work of Lagerros has been applied to the study of NEOs by Delbó (2004), Müller et al. (2004), Mueller et al. (2005), and Harris et al. (2005).

\section{The albedo distribution of NEOs}

Reliable albedo data for NEOs are available for only a very small sample of the known population. Harris \& Lagerros (2002) list results for 20 NEOs and compare results 
obtained on the basis of the three commonly used thermal models described in $\S 2$ with data from radar observations, where available, and taxonomic types. There are serious discrepancies in many cases between diameters and albedos derived from the STM and FRM and values deduced from radar observations or expected on the basis of taxonomic type. The NEATM appears to give more consistent results but also fails in at least two cases in which a default value of the model parameter, $\eta$, was used due to lack of spectral data for model fitting.

The NEATM has been used by Delbó et al. (2003) to determine the sizes and albedos of 20 NEOs observed with the Keck-I telescope on Mauna Kea. The spread of albedos was found to be very large $\left(p_{v}=0.02-0.55\right)$ although for the most part the values are consistent with those expected for the spectral types observed. A serious problem in surveys of NEO albedos is the under abundance of the darker taxonomic types $(\mathrm{C}, \mathrm{D}$, $\mathrm{P}$, etc.) in the known population due to detection bias against optically weak objects. Detection and observational biases have to be taken into account in attempts to relate surveys of samples of the NEO population to the population as a whole. In order to apply the albedo data of Delbó et al. (2003) to a study of the size distribution of NEOs, Stuart \& Binzel (2004) applied a magnitude limited de-biasing technique to produce de-biased average albedos for the taxonomic types sampled by Delbó et al. The resulting de-biased albedos were combined with a large body of taxonomic data to convert the absolute magnitude distribution of NEOs to a diameter distribution, which was used as the basis of estimates of impact rates of NEOs of different sizes. For NEOs with diameters of $1 \mathrm{~km}$ or more, Stuart \& Binzel (2004) determined an abundance of $1090 \pm 180$ and an average impact frequency on the Earth of 1 per $0.6 \pm 0.1 \mathrm{Myr}$. They obtained a value of $0.14 \pm 0.02$ for the de-biased mean albedo of the NEO population, which is higher than would be expected from comparison with the albedos in the IRAS Minor Planet Survey $(\sim 0.1$, Tedesco, 1992). In particular, the albedos of C-type NEOs appear to be some $70 \%$ higher than known C-types in the main belt, although caution is due given that this conclusion is based on a sample of only 6 NEO C-types. In any case, it is not clear why NEO C-types should have higher albedos than those observed in the main belt. Possible explanations include erroneous absolute magnitudes (could the absolute brightnesses of dark NEOs be consistently overestimated?), and the emissivity in the thermal infrared for C-type NEOs being much lower than the commonly assumed value for all asteroids of 0.9 . If the latter turned out to be the case it would offer valuable insight into the nature of C-type NEO surfaces. The uncertainty associated with the emissivity of asteroid surfaces is illustrated by the work of Walker (2003), who applied the NEATM to the IRAS Minor Planet Survey (IMPS) $12 \mu \mathrm{m}$ and $25 \mu \mathrm{m}$ fluxes for 54 asteroids with measured occultation diameters and compared those diameters with the resulting NEATM diameters. His results led Walker (2003) to conclude that the average infrared emissivity, $\varepsilon$, of the 54 IMPS asteroids is 0.79 , rather than the commonly adopted value of 0.9 . However, Walker reports that his results appear to be independent of taxonomic class. The questions of whether Walkers results also hold for NEOs, and whether emissivity depends on taxonomic class in the case of NEOs, constitute an interesting subject for future study.

Delbó et al. (2003) noted a possible trend of increasing albedo with decreasing diameter in the case of S-type NEOs with diameters below $10 \mathrm{~km}$. While the significance of this trend is not clear due to the small sample size and the possible influence of a detection bias against small, dark objects, it is consistent with the idea that collisional processing leads to reduced lifetimes for small NEOs and therefore reduced exposure to space weathering, a process that may darken the surfaces of the olivine-rich S and Q taxonomic types (Clark, Hapke, Pieters, et al. 2002). I have reviewed this apparent correlation in the light of further albedo data that have become available since the study of Delbó et al.. 
Table 1. Linear correlation coefficients for the data sets plotted in figure 1

\begin{tabular}{|c|c|c|c|}
\hline Data set & $\begin{array}{c}\text { No. } \\
\text { points }\end{array}$ & $\begin{array}{c}\text { Linear } \\
\text { correlation } \\
\text { coefft., R }\end{array}$ & $\begin{array}{c}\% \text { probability } \\
\text { that } \mathrm{R}=0\end{array}$ \\
\hline Excluding S \& Q types & 21 & -0.57 & 0.66 \\
\hline S \& Q only & 17 & -0.76 & 0.04 \\
\hline Excluding $\mathrm{S} \& \mathrm{Q}(D>0.5 \mathrm{~km}$ only $)$ & 17 & -0.24 & 36.0 \\
\hline $\mathrm{S} \& \mathrm{Q}$ only $(D>0.5 \mathrm{~km}$ only $)$ & 13 & -0.71 & 0.66 \\
\hline
\end{tabular}

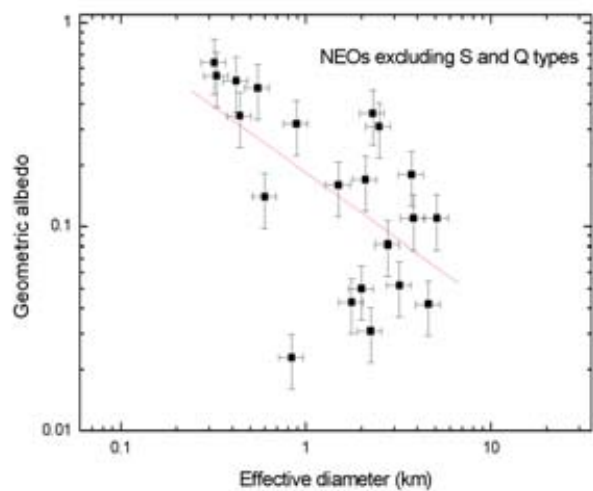

(a)

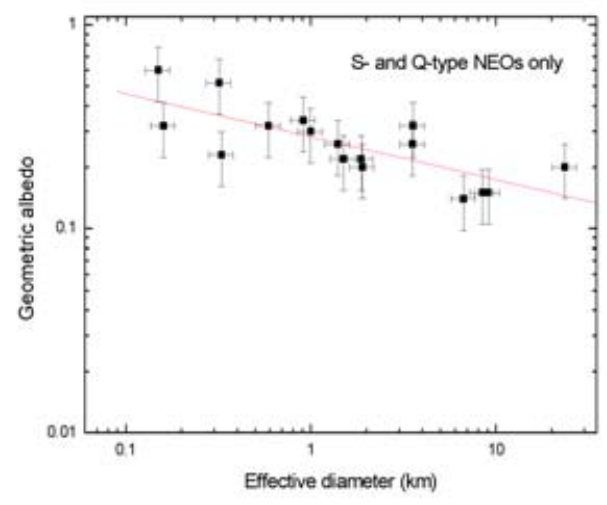

(b)

Figure 1. Plots of geometric albedo against diameter for a set of 21 NEOs excluding $\mathrm{S}$ and $\mathrm{Q}$ typers (a - left-hand frame), and for a set of $17 \mathrm{~S}$ - and Q-type NEOs (b - right-hand frame). A best-fit line is superimposed in both cases: the corresponding linear correlation coefficients are listed in table 1. The sources of the (NEATM-derived) diameter and albedo data are: Delbó et al.. (2003) and references therein, Delbó (2004), Harris et al. (2005), and Wolters et al. (2005).

In figure 1a albedo values derived on the basis of the NEATM for NEOs for which taxonomic information is available, excluding S- and Q-types, are plotted against diameter. In figure $1 \mathrm{~b}$ albedo data for S- and Q-types only are plotted. There appears to be a significant correlation in the first case (linear correlation coefficient in figure $1 \mathrm{a}=-0.57$ ), which excludes S- and Q-type NEOs, indicating that detection bias may indeed play a role (note that the correlation is much weaker if the four objects with diameters less than $0.5 \mathrm{~km}$ are excluded see table 1). However, the correlation in the second case ( $\mathrm{S}$ and $\mathrm{Q}$ only) is more significant (correlation coefficient $=-0.76$ ), suggesting that another effect in addition to detection bias is strengthening the correlation of albedo with diameter in the case of the S- and Q-type asteroids (note that in this case the correlation is only marginally weaker if the four objects with diameters less than $0.5 \mathrm{~km}$ are excluded - see table 1 ). Space weathering may be responsible for this effect, in addition to the trend to ordinarychondrite-type reflection spectra with decreasing size observed in the NEO population (Binzel, Lupishko, Di Martino, et al. 2002). It is also possible that size-dependent regolith properties, such as the size distribution of grains, may give rise to a correlation of NEO diameter with albedo. Turning to the low end of the albedo scale, a long-running debate concerns the fraction of the NEO population that has a cometary origin. Since the dynamical lifetimes of comets generally exceed their active lifetimes, there is expected 
to be a large number of dormant or extinct comets that are catalogued as asteroids. Fernández, Jewitt \& Sheppard (2005) have performed a thermal-infrared survey of 26 asteroids having comet-like orbits, including six NEOs. Diameters and geometric albedos for the target asteroids were derived on the basis of the NEATM. Fernández et al. found that some $64 \%$ of the objects had low $\left(p_{v}<0.075\right)$, comet-like albedos and considered these to be candidate dormant or extinct comets. On the basis of their results together with albedos in the literature for a further 4 NEOs, Fernández, Jewitt \& Sheppard suggest that some $53 \%$ of NEOs on comet-like orbits have comet-like albedos; assuming $7 \%$ of all NEOs are on comet-like orbits they conclude that some $4 \%$ of all NEOs are dormant or extinct comets. However, Binzel et al. (2004) estimate that $10-18 \%$ of the NEO population may be extinct comets, after correcting for observational bias against the detection of low-albedo objects in the known population of NEOs.

\section{Thermal inertia and the Yarkovsky effect}

Thermal inertia, i.e. the resistance of a material to temperature change, is defined as $(\kappa \rho c)^{0.5}$, where $\kappa$ is the thermal conductivity, $\rho$ the density and $c$ the specific heat. The surface temperature distribution of an asteroid is determined by a number of factors, including the rate of rotation, the spin-axis orientation with respect to the Sun, the surface roughness and the surface thermal inertia. An asteroid covered in a thermally-insulating dusty regolith would be expected to have a low thermal inertia. Values of thermal inertia derived for some main-belt asteroids are around 10-20 $\mathrm{Jm}^{-2} \mathrm{~s}^{-0.5} \mathrm{~K}^{-1}$ (Müller \& Lagerros 1998). The thermal inertia of the lunar surface is some $50 \mathrm{Jm}^{-2} \mathrm{~s}^{-0.5} \mathrm{~K}^{-1}$ (see for example Spencer, Lebofsky \& Sykes 1989) and that of bare rock about $2500 \mathrm{Jm}^{-2} \mathrm{~s}^{-0.5} \mathrm{~K}^{-1}$ (Jakosky 1986). The surface temperature distribution of a body with low thermal inertia rotating slowly, with the Sun above its equator, has a prominent peak near the sub-solar point. The amplitude of the equatorial temperature distribution decreases with increasing surface thermal inertia or rotation rate.

Areas in which knowledge of thermal inertia is of crucial importance are the design of asteroid lander missions, for which diurnal thermal cycling can affect the lifetime and performance of scientific instruments, and the calculation of the gradual orbital drift of small asteroids due to the anisotropic emission of thermal-infrared photons, known as the Yarkovsky effect. The very small force that results from the anisotropic radiation of absorbed solar energy has been invoked to explain the apparent long time scale for the delivery of collisional fragments into orbital resonances in the main belt, from which they are injected into near-Earth orbits. The Yarkovsky effect has a significant influence on the orbital evolution of potentially hazardous near-Earth objects (PHOs). For example, considering two extreme but plausible values of thermal inertia, Giorgini et al. (2002) showed that the uncertainty in the Yarkovsky effect alone leads to an uncertainty of $83 \times 10^{6} \mathrm{~km}$ in the along-track position of the PHO 1950 DA just before the time of nominal orbit intersection with the Earth in the year 2880. This example highlights the importance of investigations of the thermal properties of NEO surfaces for calculations of collision probabilities of potentially hazardous objects.

The derivation of a reliable value for the surface thermal inertia of an asteroid, especially a small object or $\mathrm{NEO}$, is a challenging task that requires accurate photometry of the thermal continuum emission and a detailed thermal model. However, some information on thermal inertia can be obtained from the apparent color temperature of an asteroid observed at a low solar phase angle. The model parameter, $\eta$, in the NEATM can be used as a measure of the deviation of the observed colour temperature from that expected on the basis of the STM (in which $\eta=1$ for a smooth sphere, or less than 1 in 
the case of beaming due to roughness). As explained above, the sub-solar temperature decreases with increasing thermal inertia for an object rotating with the Sun on its equator. A low sub-solar temperature observed at a low phase angle will be reflected in a relatively high best-fit value of $\eta$ (cf. 2.2), so high values of $\eta$ at low phase angles are indicative of high thermal inertia. In a set of 17 NEOs in the size range $0.6-25 \mathrm{~km}$ for which $\eta$ has been determined by thermal continuum fitting, Delbó et al. (2003) noted that $\eta$ appears to increase with phase angle and that the only objects displaying values of $\eta$ significantly higher than unity were observed at solar phase angles exceeding $35^{\circ}$. An updated plot of $\eta$ versus solar phase angle for a set of NEOs with adequate multi-filter photometric data to enable $\eta$ to be derived via NEATM fitting is shown in figure 2 ; values of $\eta$ resulting from applying the NEATM to synthetic fluxes from perfect STM (zero thermal inertia and no beaming) and FRM (fast rotation/high thermal inertia, Sun on equator) asteroids are traced by the dashed straight line and dotted curve, respectively. Note that the $\eta$

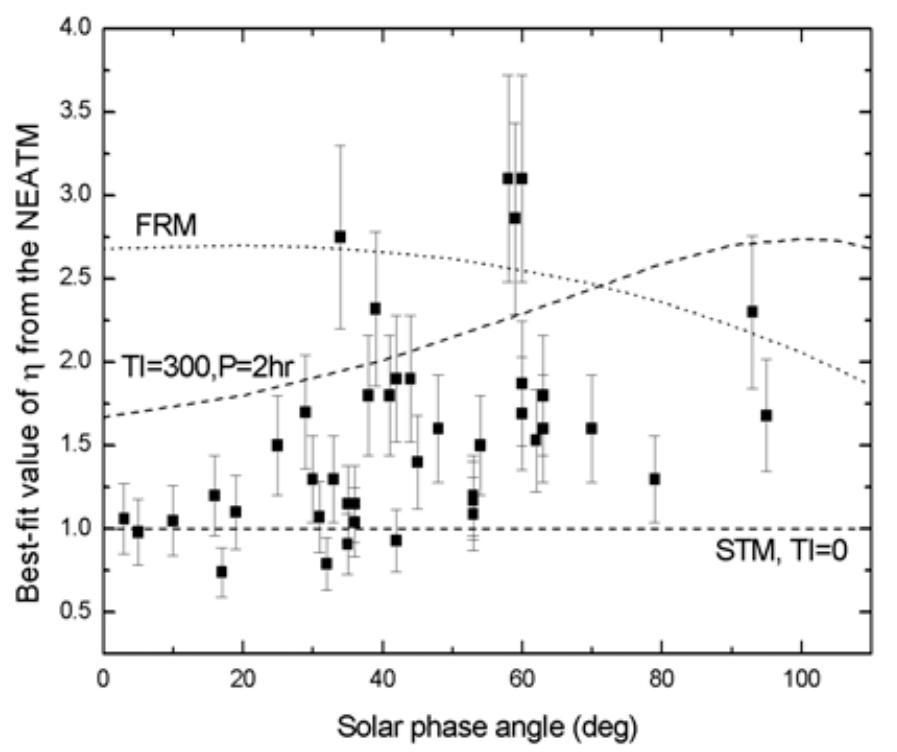

Figure 2. Plot of the best-fit $\eta$ value from the NEATM against solar phase angle for a set of NEOs with adequate multi-filter photometric data to enable $\eta$ to be derived via spectral fitting (the data set includes multiple values of $\eta$ for some objects observed at more than one phase angle). The error bars represent a conservative $20 \%$ uncertainty, which is based on the reproducibility of $\eta$ for those objects for which more than one measurement from independent data sets is available. The dashed line at $\eta=1$ represents a lower limit given by an STM-type asteroid (zero thermal inertia or spin axis pointing at the Sun) with a smooth surface. If beaming due to surface roughness is present, $\eta$ may be lower than unity at low phase angles. The dotted curve labeled FRM represents an upper limit for a population of FRM-type asteroids (fast rotation/high thermal inertia surface, e.g. bare rock). The dashed curve represents an upper limit for a population of spherical, smooth asteroids at $1 \mathrm{AU}$ from the Sun with thermal inertia $=\mathrm{Jm}^{-2} \mathrm{~s}^{-0.5} \mathrm{~K}^{-1}$ and rotation periods longer than $2 \mathrm{hr}$ (note: the presence of surface roughness could increase $\eta$ above this lever at high phase angles). In particulr, the fact that the points cluster around the low-thermal-inertia limit at low phase angles (i.e. less than $30^{\circ}$ ) suggests there is a lack of NEOs with high thermal inertia in the set of objects observed to data. For data sources see captions to figure 1 (some additional unpublished data have been included). 
value of a fast-rotating asteroid with high thermal inertia decreases as the angle between the spin axis and the solar direction decreases. The temperature distribution of an FRM asteroid with its spin axis pointing towards the Sun is identical to that of the STM. So for a population of FRM-type asteroids (fast rotation/high thermal inertia surface, e.g. bare rock) with randomly oriented spin axes, the dashed curve labeled FRM represents an upper limit for $\eta$. Likewise, for a population of spherical, smooth asteroids with thermal inertia $=300 \mathrm{Jm}^{-2} \mathrm{~s}^{-0.5} \mathrm{~K}^{-1}$, rotation periods longer than $2 \mathrm{hr}$ (true for nearly all NEOs with known periods) and random spin-axis orientations, the $\eta$ value distribution in figure 2 would be bounded by the dashed STM line and the dashed curve labeled $T I=300$, $P=2 \mathrm{hr}$. While the data set is small, the distribution of $\eta$ values in figure 2 appears to favor the latter case, rather than a population consisting largely of high thermal inertia objects. In particular, the absence of high values of $\eta$ at low phase angles suggests that objects with high thermal inertia, in the size range investigated, may be relatively rare (see $\S 5$ ). The presence of a significant beaming effect due to surface roughness would reduce $\eta$ below unity at low phase angles. At high phase angles the beaming effect, which enhances thermal emission at low phase angles, tends to reduce the amount of thermal emission observed and the apparent color temperature. Therefore at high phase angles both surface roughness and thermal inertia lead to increased values of $\eta$ and unraveling their contributions requires sophisticated thermal modeling.

Note that for fixed $\eta$ thermal inertia is proportional to $P^{0.5}$, where $P$ is the rotation period (see, for example, Spencer, Lebofsky \& Sykes 1989). Therefore the dashed upper limit curve in figure 2 labeled $T I=300, P=2 \mathrm{hr}$ also holds for a population of NEOs in which each object has a typical rotation period of $P=6 \mathrm{hr}$ and thermal inertia not exceeding $520 \mathrm{Jm}^{-2} \mathrm{~S}^{-0.5} \mathrm{~K}^{-1}$. Delbó (2004) has analyzed the $\eta$-value distribution shown in figure 2 with the aid of a thermophysical model that includes physical descriptions of both thermal inertia and beaming resulting from a uniform distribution of craters, and takes account of the assumed random distribution of spin axes. Assuming all objects have the same thermal inertia, and taking account of their known rotation rates, Delbó varied the thermal inertia and the degree of surface roughness until he obtained the best match to the $\eta$ versus solar phase angle distribution; his resulting best-fit value of thermal inertia is $500 \pm 100 \mathrm{Jm}^{-2} \mathrm{~s}^{-0.5} \mathrm{~K}^{-1}$.

Three estimates of thermal inertia obtained via thermophysical modeling for individual NEOs for which shape models are available are given in table 2, together with some values derived for main-belt asteroids by Müller \& Lagerros (1998).

In summary, the results reviewed here suggest that the appropriate values of thermal inertia to adopt in calculations of the Yarkovsky effect on NEOs are an order of magnitude

Table 2. Comparison of asteroid thermal inertia determinations from thermophysical modeling

\begin{tabular}{lccl}
\hline \multicolumn{1}{c}{ Object } & $D_{\text {eff }}(\mathrm{km})$ & $\begin{array}{c}\text { Thermal inertia } \\
\left(\mathrm{Jm}^{-2} \mathrm{~s}^{-0.5} \mathrm{~K}^{-1}\right)\end{array}$ & Source \\
\hline (1) Ceres & & 10 & Müller \& Lagerros (1998) \\
(4) Vesta & 523 & 25 & Müller \& Lagerros (1998) \\
(2) Pallas & 530 & 10 & Müller \& Lagerros (1998) \\
(3) Juno & 2400 & 5 & Müller \& Lagerros (1998) \\
(532) Herculina & 218 & 15 & Müller \& Lagerros (1998) \\
(433) Eros (NEO) & 17.5 & $\sim 150$ & Mueller et al. $(2005)$ \\
(1580) Betulia (NEO) & $4.57 \pm 0.46$ & $\sim 180$ & Harris et al. $(2005)$ \\
(25143) Itokawa (NEO) & $\sim 0.3$ & $\sim 350$ & Mueller et al. $(2005)$ \\
Bare rock & & $\sim 2500$ & Jakosky (1986) \\
\hline
\end{tabular}


or more higher than those derived for main-belt asteroids, but much less than that of bare rock.

\section{Where are the asteroids with bare rock surfaces?}

The C-type asteroid Betulia is significant in being the first NEO for which thermalinfrared observations (Lebofsky, Veeder, Lebofsky, et al. 1978) indicated a surface of high thermal inertia. In contrast to all asteroids previously observed in the thermal infrared, the measured infrared fluxes of Betulia, together with the size estimates from radar and polarimetric observations, appeared to be inconsistent with the standard thermal model, which assumes no or very little thermal inertia. Lebofsky et al. (1978) found that a thermal model having the characteristics of bare rock, similar to the FRM, was required to give a size consistent with that derived from polarimetry and radar observations. The diameter resulting from their model is $7.5 \pm 0.34 \mathrm{~km}$. Harris et al. (2005) applied a thermophysical model developed by M. Mueller (paper in preparation) to recent thermalinfrared flux measurements of Betulia obtained at the NASA Infrared Telescope Facility (IRTF) and derived a diameter of $4.57 \pm 0.46 \mathrm{~km}$ and an albedo of $p_{v}=0.077 \pm 0.015$ (figure 3). The resulting thermal inertia is around $180 \mathrm{Jm}^{-2} \mathrm{~s}^{-0.5} \mathrm{~K}^{-1}$, or some three times the lunar value. This value of thermal inertia is less than $10 \%$ of that expected for a bare-rock surface and implies that the surface of Betulia has a significant thermallyinsulating regolith, in contrast to the conclusions of earlier work. The reason why the more recent results for the size and thermal inertia of Betulia differ markedly from the earlier results discussed by Lebofsky et al. (1978) is not clear. Betulia is well known for its unusual lightcurve, the amplitude and form of which changes dramatically with

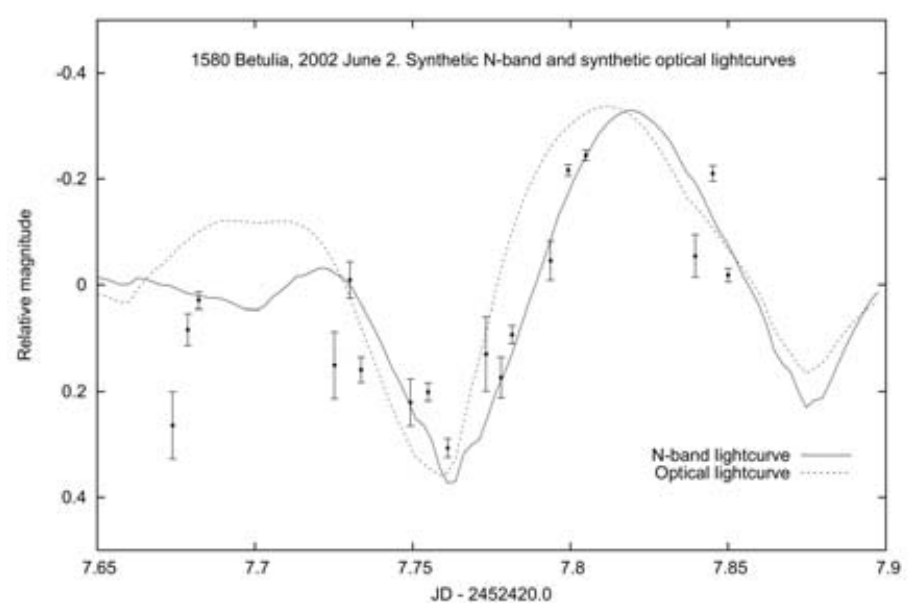

Figure 3. Synthetic thermal infrared (N-band) and synthetic optical lightcurves for (1580) Betulia generated using a thermophysical model (incorporating a shape model) for the observing geometry of 2002 June 2. Points with $1-\sigma$ statistical error bars corresponding to the measured N-band fluxes are superimposed. The zero-magnitude level of the N-band lightcurve refers to the mean flux level; the zero-magnitude level in the case of the N-band measurements refers to the NEATM thermal contiuum fit to the lightcurve-corrected data. No relative manual adjustment to the N-band synthetic lightcurve or measurement points were made. The shape model and thermophysical model generate optical and thermal-infrared lightcurves that are in good agreement with the observations. The value of thermal inertia resulting from the thermophysical model fit to the data is $180 \mathrm{Jm}^{-2} \mathrm{~S}^{-0.5} \mathrm{~K}^{-1}$, which is much less than the expected for a surface of bare rock. For further deatils see Harris et al. (2005). 
changing solar phase angle, probably as a result of a highly irregular shape and/or unusual topographic features (e.g. Tedesco, Drummond, Candy, et al. 1978). It is important to bear the unusual nature of Betulia in mind when interpreting observational data with the aid of standard optical, thermal-infrared, and other techniques. It is interesting to note that if the data of Lebofsky et al. (1978) are analyzed on the assumption of low thermal inertia, a similar (smaller) diameter is obtained to that derived by Harris et al. (2005). So the two sets of infrared data appear to be consistent if a relatively low thermal inertia model is used, but not if a model with the thermal characteristics of bare rock is used. On the other hand, the earlier polarimetry and radar observations appear to support a larger size. Further observations of Betulia will be required to resolve these issues. In any case, observations of unusual objects such as Betulia are particularly valuable for exploring the limits of applicability and reliability of the various analysis techniques applied to asteroid data.

While the absence of high $\eta$ values at low solar phase angles in figure 2 suggests that in general NEOs with high thermal inertia are lacking in the population observed to date, it should be noted that some of the relatively high $\eta$ values evident in figure 2 might be at least partly due to unusually high thermal inertia. However, as mentioned in $\S 4$, surface roughness confuses the issue at large phase angles where it can also lead to increased $\eta$ values. Judging from their high $\eta$ values measured at moderate phase angles, the best candidates for unusually high thermal inertia surfaces amongst the objects studied to date appear to be $2002 \mathrm{HK}_{12}$ (Wolters, Green, McBride, et al. 2005), with $\eta=2.75$ at a phase angle of $33^{\circ}$ and (2100) Ra-Shalom (Delbó, Harris, Binzel, et al. 2003), with $\eta=2.32$ at a phase angle of $39^{\circ}$ (see figure 2 ).

There is good reason to expect that regolith-free surfaces will be found amongst very small, rapidly rotating NEOs. While confirmation of a size-dependence of thermal inertia must await the collection of data for many more objects in the sub-km size range, it seems likely that the very weak surface gravities of such objects, especially if they rotate rapidly, would be unable to retain significant thermally-insulating surface debris from impacts, unless some other effect is causing material such as dust to adhere to their surfaces. One object that promises to throw some light on this question is the NEO (54509) $2000 \mathrm{PH}_{5}$, which is in a dynamically interesting Sun-Earth horseshoe orbit. The size of $2000 \mathrm{PH}_{5}$ is $100-200 \mathrm{~m}$ and its rotation period is just $0.2 \mathrm{hr}$ (from P. Pravec: http://sunkl.asu.cas.cz/p̃pravec/). Current observational programs should provide information on the thermal inertia of $2000 \mathrm{PH}_{5}$ and whether anisotropic thermal emission is gradually modifying its rotation rate (the YORP effect).

\section{How reliable are the results from thermal models?}

Unfortunately at the present time very few ground-truth data are available on the sizes, albedos, thermal inertia and surface roughness of asteroids to compare with results from astronomical observations and thermal modeling. It is therefore very difficult to calibrate the models against the characteristics of known objects. Even the sophisticated thermophysical models can do no better than adopt simplified and idealized descriptions of thermal inertia and surface roughness. For example, it is not clear that the effects of surface roughness on very small, irregular bodies can be adequately described by a uniform distribution of hemispherical craters (cf. $\S 2$ ). A more sophisticated treatment may be required that takes account of intermediate-scale topographical structure below the resolution of the shape models. While sizes and albedos derived from thermophysical models should be more reliable than those from the simpler models outlined in $\S 2$, the 


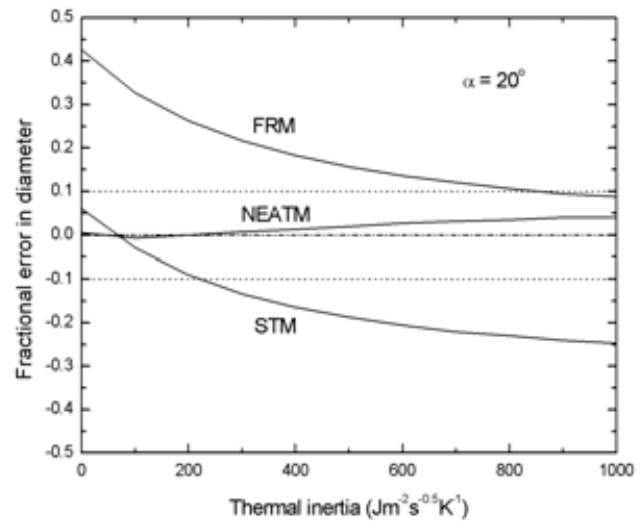

(a)

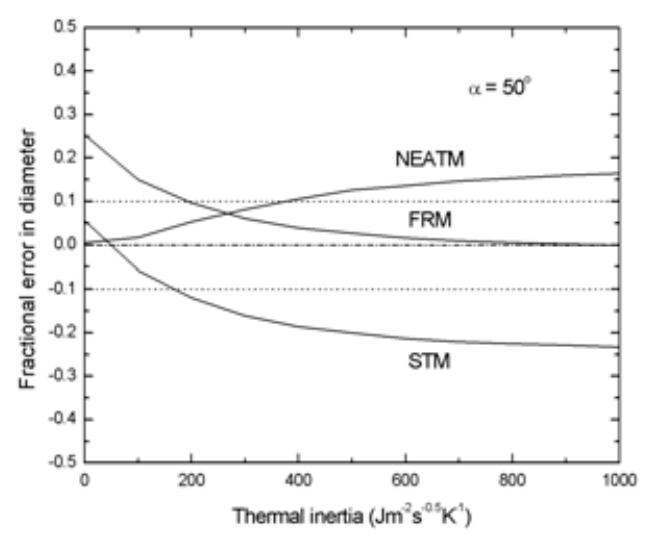

(b)

Figure 4. Plots of the performances of the STM, NEATM, and FRM against thermal inertia for solar phase angles of $\alpha=20^{\circ}$ and $\alpha=50^{\circ}$. Test data were generated using a smooth spherical model incorporating the effects of thermal inertia. In the STM the beaming parameter, $\eta$, was set to unity. The model asteroid had a rotation period of $6 \mathrm{hr}$ and the sub-solar and sub-Earth latitudes were zero; other parameters were chosen to be typical of NEOs. The sense of the phase angle is such that the cooler, morning side of the asteroid was viewed. The small positive error at thermial inertia $=0$ in the case of the STM results from the use of the phase coefficient of $0.01 \mathrm{mag} / \mathrm{deg}(\mathrm{see} \S 2)$

corresponding values of thermal inertia and surface roughness should be treated with caution until more ground-truth data are available from space missions.

The simpler models based on spherical geometry serve the purpose of giving reasonable estimates of size and albedo in the absence of knowledge of an asteroids shape and spin vector. The accuracy obtainable with the simpler models depends on the circumstances in which they are applied. Given the range of values of thermal inertia for main-belt asteroids and NEOs listed in table 2, it is possible to gain insight into the limits of applicability of the simpler models.

To provide an illustration, synthetic flux values were generated using a smooth spherical model that incorporates the effects of thermal inertia by means of a one-dimensional vertical heat-transfer algorithm. The model asteroid had a rotation period of $6 \mathrm{hr}$ and the sub-solar and sub-Earth latitudes were zero; other parameters were chosen to be typical of NEOs. Values of diameter were derived from the synthetic fluxes using the STM (with $\eta=1.0$, i.e. smooth surface), NEATM and FRM outlined in $\S 2$ for various values of thermal inertia and phase angle, and the fractional error in the diameter calculated in each case. Figure 4 shows the dependence of the fractional error in diameter on thermal inertia for solar phase angles, $\alpha$, of $20^{\circ}$ and $50^{\circ}$ (n.b. the sense of the phase angle is such that the cooler, morning side of the asteroid was viewed). For low values of thermal inertia, typical of main-belt asteroids (see table 2) and the Moon, the STM and the NEATM both perform well at both values of phase angle, whereas the FRM grossly overestimates the diameter. At $\alpha=20^{\circ}$ the NEATM performs best over the full range of thermal inertia, while for thermal inertia exceeding $100 \mathrm{Jm}^{-2} \mathrm{~s}^{-0.5} \mathrm{~K}^{-1}$ the STM underestimates the diameter to an extent that worsens with increasing thermal inertia. On the other hand the performance of the FRM improves with increasing thermal inertia, as expected. At $\alpha=50^{\circ}$ the performance of the FRM is better than that of the NEATM for 


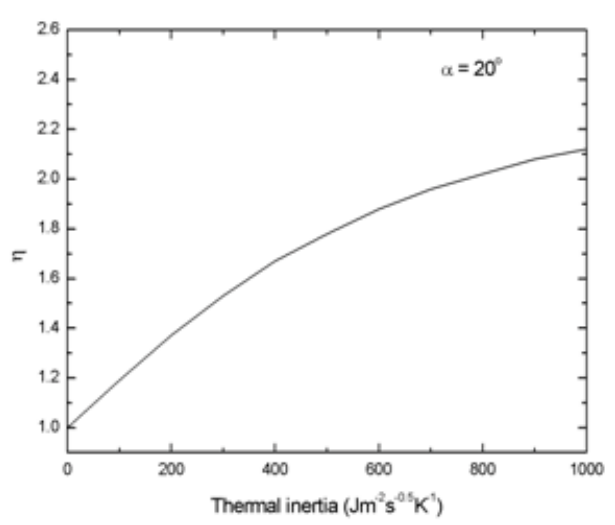

(a)

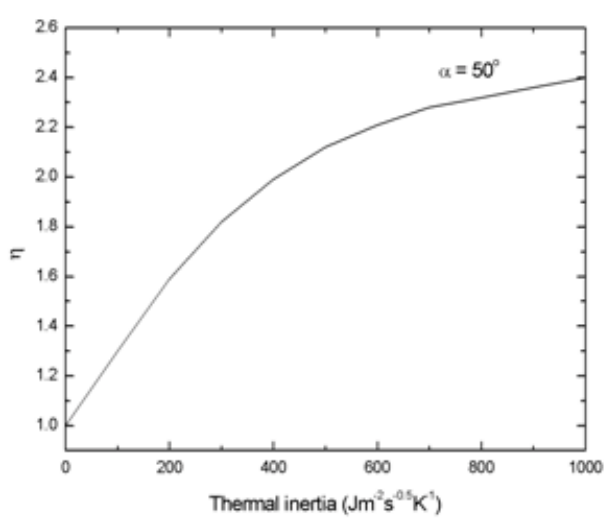

(b)

Figure 5. Plots of the NEATM model parameter $\eta$ corresponding to the NEATM solutions plotted in figure 4 .

values of thermal inertia above $250 \mathrm{Jm}^{-2} \mathrm{~s}^{-0.5} \mathrm{~K}^{-1}$, although the NEATM error remains below $15 \%$ over most of the thermal inertia range. The NEATM overestimates the diameter (and therefore underestimates the albedo) due to the fact that it ignores thermal emission from the night side of the object, a problem that worsens with increasing phase angle and thermal inertia.

It should be noted that the plots of figure 4 represent worst cases for the NEATM and the STM in the sense that the errors were calculated for zero sub-solar latitude. For other values of sub-solar latitude (i.e. non-perpendicular inclinations of the spin-axis to the solar direction) the errors due to thermal inertia will be lower: as mentioned above, in the limit of sub-solar latitude $=90^{\circ}$, i.e. spin axis pointing towards the Sun, the temperature distribution of an FRM-type object is identical to that of the STM, i.e. an object with zero thermal inertia.

Values of $\eta$ corresponding to the NEATM solutions plotted in figure 4 are shown in figure 5. There is a strong dependence of $\eta$ on thermal inertia, which demonstrates why the STM, in which $\eta$ is held constant, is not applicable to asteroids with significant thermal inertia.

\section{Summary and discussion}

Observations of asteroids in the thermal infrared are the main source of information on their sizes, albedos, and thermal inertia. Knowledge of these parameters is crucial for many aspects of asteroid research, including studies of the size distribution and impact hazard of NEOs, the taxonomic composition of populations of asteroids, the physical properties of asteroid surfaces and regolith, and calculations of the magnitude of the Yarkovsky effect. To derive physical properties from thermal-infrared data a thermal model of the asteroid is required. Commonly used models based on spherical geometry can provide acceptable results, especially when combined with knowledge of an objects optical lightcurve, but have to be applied with care, especially in the case of small objects such as NEOs. Modern computers enable the use of sophisticated thermophysical models based on realistic shapes that incorporate physical descriptions of thermal inertia and 
surface roughness. However, some prior knowledge of an objects rotation vector and shape is a prerequisite for the use of thermophysical models.

A very broad range of albedos is observed amongst the NEO population. There is some evidence for the effects of space weathering, or some other surface phenomenon, leading to smaller S- and Q-type objects having higher albedos. However, detection bias against small, dark objects can also give rise to such a correlation. While the present data set suggests that detection bias alone cannot explain the correlation, a final resolution of this question must await the availability of a much larger data set.

Insight into the thermal inertia of asteroids can be gained from a study of the variation of the model parameter $\eta$ with solar phase angle. The parameter $\eta$ in the NEATM is a measure of the deviation of the observed color temperature from that expected for a smooth sphere with zero thermal inertia. Results suggest that the thermal inertia values of most NEOs observed to date are well below the value for bare rock $(\sim 2500$ $\left.\mathrm{Jm}^{-2} \mathrm{~s}^{-0.5} \mathrm{~K}^{-1}\right)$. Values of thermal inertia for a few individual NEOs derived on the basis of thermophysical modeling are in the range $100-400 \mathrm{Jm}^{-2} \mathrm{~s}^{-0.5} \mathrm{~K}^{-1}$, consistent with surfaces largely covered in thermally-insulating regolith or dust but an order of magnitude higher than values derived for large main-belt asteroids. Thermal inertia is an important parameter in the calculation of the orbital drift of potentially hazardous asteroids due to the Yarkovsky effect. Further observations are required to probe smaller objects and to determine the extent to which the presence of thermally-insulating surface material, and therefore thermal inertia, is size dependent.

The application of sophisticated thermophysical models incorporating information on shape and spin vector to observational data has the potential to teach us a great deal about asteroid surface properties. Unfortunately information on topographical structure analogous to hills, valleys, ridges, etc., is rarely available, so the modeling of surface structure is normally limited to an idealized uniform distribution of craters represented by hemispheres, or sections thereof. The over-simplification of surface structure in thermophysical models may seriously limit their accuracy in the case of NEOs, which are often observed at large phase angles at which shadowing effects are more significant and measured fluxes are presumably strongly dependent on topographical structure, in addition to cratering. The influence of intermediate-scale surface structure on the thermophysical modeling of asteroids is an important area for future study.

\section{References}

Binzel, R.P., Lupishko, D., Di Martino, M., Whiteley, R.J., \& Hahn, G.J. 2002, in: W. Bottke, A. Cellino, P. Paolicchi \& R.P. Binzel (eds.), Asteroids III, (Tucson: Univ. Arizona Press), p. 255

Binzel, R.P., Rivkin, A.S., Stuart, J.S., Harris, A.W., Bus, S.J., \& Burbine, T.H. 2004, Icarus 170,259

Clark, B.E., Hapke, B., Pieters, C., \& Britt, D. 2002, in: W. Bottke, A. Cellino, P. Paolicchi \& R.P. Binzel (eds.), Asteroids III, (Tucson: Univ. Arizona Press), p. 585

Delb, M. 2004, Doctoral thesis, Freie Universität Berlin.

Delbó, M. \& Harris, A.W. 2002, Meteoritics and Planet. Sci. 37, 1929

Delbó, M., Harris, A.W., Binzel, R.P, Pravec, P., \& Davies, J.K. 2003, Icarus 166, 116

Fernández, Y.R., Jewitt, D.C., \& Sheppard, S.S. 2005, A.J. in press.

Giorgini, J.D., Ostro, S.J., Benner, L.A.M., Chodas, P.W., Chesley, S.R., Hudson, R.S., Nolan, M.C., Klemola, A.R., Standish, E.M., Jurgens, R.F., Rose, R., Chamberlin, A.B., Yeomans, D.K., \& Margot, J.-L. 2002, Science 296, 132

Harris, A.W. 1998, Icarus 131, 291

Harris, A.W. \& Lagerros, J.S.V. 2002, in: W. Bottke, A. Cellino, P. Paolicchi \& R.P. Binzel (eds.), Asteroids III, (Tucson: Univ. Arizona Press), p. 205 
Harris A.W., Mueller, M., Delbó, M., \& Bus, S.J. 2005, Icarus in press.

Jakosky B.M. 1986, Icarus 66, 117

Kaasalainen, M., and 21 colleagues 2004, Icarus 167, 178

Lebofsky, L.A. \& Spencer, J.R. 1989, in: R. P. Binzel, T. Gehrels \& M. S. Matthews (eds.), Asteroids II, (Tucson: Univ. Arizona Press), p. 128

Lebofsky, L.A., Sykes, M.V., Tedesco, E.F., Veeder, G.J., Matson, D.L., Brown, R.H., Gradie, J.C., Feierberg, M.A., \& Rudy, R.J. 1986, Icarus 68, 239

Lebofsky, L.A., Veeder, G.J., Lebofsky, M.J., \& Matson, D.L. 1978, Icarus 35, 336

Mueller, M., Delbó, M., Di Martino, M., Harris, A.W., Kaasalainen, M., \& Bus, S.J. 2005, ASP Conference Series, (submitted Dec. 2004)

Müller, T.G. \& Lagerros, J.S.V. 1998, Astron. Astrophys. 338, 340

Müller, T.G., Sterzik, M.F., Schütz, O., Pravec, P., \& Siebenmorgen, R. 2004, Astron. Astrophys. 424,1075

Spencer, J.R., Lebofsky, L.A., \& Sykes, M.V. 1989, Icarus 78, 337

Stuart, J.S. \& Binzel, R.P. 2004, Icarus 170, 295

Tedesco, E.F. (ed. 1992, The IRAS minor planet survey Tech. Rep. PL-TR-92-2049. Phillips Lab., Hanscom Air Force Base, MA.

Tedesco, E., Drummond, J., Candy, M., Birch, P., Nikoloff, I., \& Zellner, B. 1978, Icarus 35, 344

Walker, R.G. 2003, Bulletin of the American Astronomical Society 35, abstract 34.19

Wolters, S.D., Green, S.F., McBride, N., \& Davies, J.K. 2005, Icarus 175, 92 